\title{
Some Analytic Results for the Study of Broadband Noise Radiation from Wings, Propellers and Jets in Uniform Motion*
}

\author{
F. Farassat and J. Casper \\ NASA Langley Research Center \\ Hampton, Virginia
}

\begin{abstract}
Alan Powell has made significant contributions to the understanding of many aeroacoustic problems, in particular, the problems of broadband noise from jets and boundary layers. In this paper, some analytic results are presented for the calculation of the correlation function of the broadband noise radiated from a wing, a propeller, and a jet in uniform forward motion. It is shown that, when the observer (or microphone) motion is suitably chosen, the geometric terms of the radiation formula become time independent. The time independence of these terms leads to a significant simplification of the statistical analysis of the radiated noise, even when the near field terms are included. For a wing in forward motion, if the observer is in the moving reference frame, then the correlation function of the near and far field noise can be related to a space-time cross-correlation function of the pressure on the wing surface. A similar result holds for a propeller in forward flight if the observer is in a reference frame that is attached to the propeller and rotates at the shaft speed. For a jet in motion, it is shown that the correlation function of the radiated noise can be related to the space-time crosscorrelation of the Lighthill stress tensor in the jet. Exact analytical results are derived for all three cases. For the cases under present consideration, the inclusion of the near field terms does not introduce additional complexity, as compared to existing formulations that are limited to the far field.
\end{abstract}

\section{Introduction}

Alan Powell's career in acoustics spans a period of more than fifty years. He has made important contributions to the twentieth century's major areas of acoustic research, including random vibration, edge tones, jet noise and boundary layer noise. Alan Powell was one of the first researchers to recognize the importance of trailing edge noise [1]. Moreover, he has kept up admirably with developments in all of these areas. His publications convey a deep understanding of the physics of acoustic phenomena. He writes clearly and explains ideas lucidly with a masterful application of analytical techniques. It is with great pleasure that the authors dedicate this paper to Alan Powell for his lifetime achievements. He is the Grand Teacher to us all.

There is a long history of research in broadband noise radiation from jets and surfaces (see the references in many chapters of [2]). Today's aeronautical problems that require

* Published in International Journal of Aeroacoustics, Vol. 2, No. 3 \& 4, 2003, pp. 335 - 350. 
broadband noise prediction are found to present some of the most challenging aeroacoustic problems to date. It is fair to say that the acoustic analogy has played a major role in the analysis of such problems. Much insight and understanding have resulted from such analysis $[3,4,5,6]$. However, the lack of sufficient measured data, as well as the complexity of the problems themselves, has caused researchers to make assumptions that simplify their analyses and render approximate formulations. Two of the most common simplifying assumptions are that of an observer well into the far field and a source occurring at low Mach number. The analysis presented herein will demonstrate a method by which both of these assumptions can be relaxed. Furthermore, the authors feel that there is a need to remove some or all of the approximations that are commonly made in broadband noise prediction.

In order to predict a random signal that results from a broadband source, it is logical to develop an acoustic formulation that is based on statistical analysis. Previous authors have derived important results with the use of statistical analysis, e.g., [2, 3, 4, 5, 6]. Furthermore, because of the current interest in airframe noise, recent reports on statistically based research are appearing in the literature, e.g., [7]. To the present authors' knowledge, there are no published results that are similar to those derived herein.

In the present paper, some analytic results are presented for the calculation of broadband noise radiation from wings, propellers and jets in uniform rectilinear motion. The objective is to obtain exact formulations for such calculations, with the assumption that some statistical properties of the flow are known on the surface of the wing or propeller blade, or within the volume of the jet. Because exact results are desired, any near field terms that occur in the derivation are included. No assumption is made on the source speed, other than a restriction to subsonic motion. It is shown that the inclusion of the near field terms does not add more complexity to the derivation of the final formulations. It is also important to realize that the inclusion of the near field terms does not further complicate numerical algorithms that are used for noise calculations.

The following section describes the analytical approach to the three formulations derived in this work. Each derivation begins with an appropriately chosen form of Lighthill's acoustic analogy [8], with its usual solution, i.e., with respect to an observer (or microphone) that is fixed with respect to the medium at rest. A suitably chosen observer motion then results in the time independence of all the geometric terms in the radiation formula. Such a result greatly simplifies a statistical analysis of the radiated noise, even when the near field terms are retained. The autocorrelation of the acoustic pressure can then be directly related to the cross-correlation of the surface pressure in a simple form that can be interpreted physically.

In Section 3, the analytical method described above is first applied to a wing in uniform rectilinear subsonic motion with fluctuating surface pressure. For an observer fixed to the undisturbed medium, the acoustic pressure is given by the solution of the loading term of the Ffowcs Williams-Hawkings (FW-H) equation [9]. The closed form solution to this equation is available and is the foundation for the analysis. If the observer is relocated to a frame of reference that moves with the wing, then many of the terms in the solution become time independent, yielding a simpler result that avails itself to a statistical formulation. A correlation function of the acoustic pressure is then derived 
that is valid in the near and far fields. The resulting exact formulation can be simplified by making various geometric or physical approximations for the derivation of other useful results.

In Section 4, the method by which the correlation function is derived in Section 3 is extended to a propeller in uniform forward motion by locating the observer in a reference frame that rotates at the shaft speed of a propeller. The time independent functions appearing in the acoustic pressure correlation function for a propeller can be computed numerically, and not analytically as in the case of a wing in motion.

The analysis begins similarly for a jet in forward flight in Section 5, with an exact result for the acoustic pressure at an observer position fixed to the undisturbed medium. The transfer of the observer to a frame moving with the jet results in many time independent terms. The acoustic pressure correlation function is found by the same procedure that is used for a wing or a propeller in motion. Some of the consequences of the derived results are discussed.

\section{Analytical Approach}

The time domain approach used in the present work is based on Lighthill's acoustic analogy [8]. The governing equations are always solved in a reference frame that is fixed to the undisturbed medium. The space-time variables $(\overrightarrow{\boldsymbol{x}}, t)$ are associated with this fixed reference frame. In addition, the analysis requires an observer in a moving frame of reference with associated space-time variables $\left(\overrightarrow{\boldsymbol{x}}_{0}, t\right)$. Spatial locations within these two reference frames are related by the coordinate transformation $\overrightarrow{\boldsymbol{x}}=\overrightarrow{\boldsymbol{x}}\left(\overrightarrow{\boldsymbol{x}}_{0}, t\right)$. The acoustic pressure in the frame fixed to the undisturbed medium is denoted by $p^{\prime}(\overrightarrow{\boldsymbol{x}}, t)$. In the moving frame, the acoustic pressure is $\tilde{p}^{\prime}\left(\overrightarrow{\boldsymbol{x}}_{0}, t\right)$. Note that $p^{\prime}(\overrightarrow{\boldsymbol{x}}, t)$ and $\tilde{p}^{\prime}\left(\overrightarrow{\boldsymbol{x}}_{0}, t\right)$ are represented by different analytical expressions and, therefore, the present authors chose to use different symbols for these two functions.

All of the present derivations begin with an equation of the form

$$
\square^{2} p^{\prime}(\overrightarrow{\boldsymbol{x}}, t)=Q(\overrightarrow{\boldsymbol{x}}, t)
$$

where $\square^{2}$ represents the linear wave operator, and $Q(\vec{x}, t)$ is the appropriate source distribution for a given problem. Eq. (1) represents the propagation of sound to an observer in the reference frame that is fixed to the undisturbed medium. An analytic expression is now desired for $\tilde{p}^{\prime}\left(\overrightarrow{\boldsymbol{x}}_{0}, t\right)$, the acoustic pressure perceived by an observer in a moving frame of reference.

Fig. 1 schematically shows two paths to the desired solution in the moving reference frame. Taking the first path, along the solid line, leads immediately to an equation of the form

$$
\mathcal{L} \tilde{p}^{\prime}\left(\vec{x}_{0}, t\right)=\tilde{Q}\left(\vec{x}_{0}, t\right)
$$




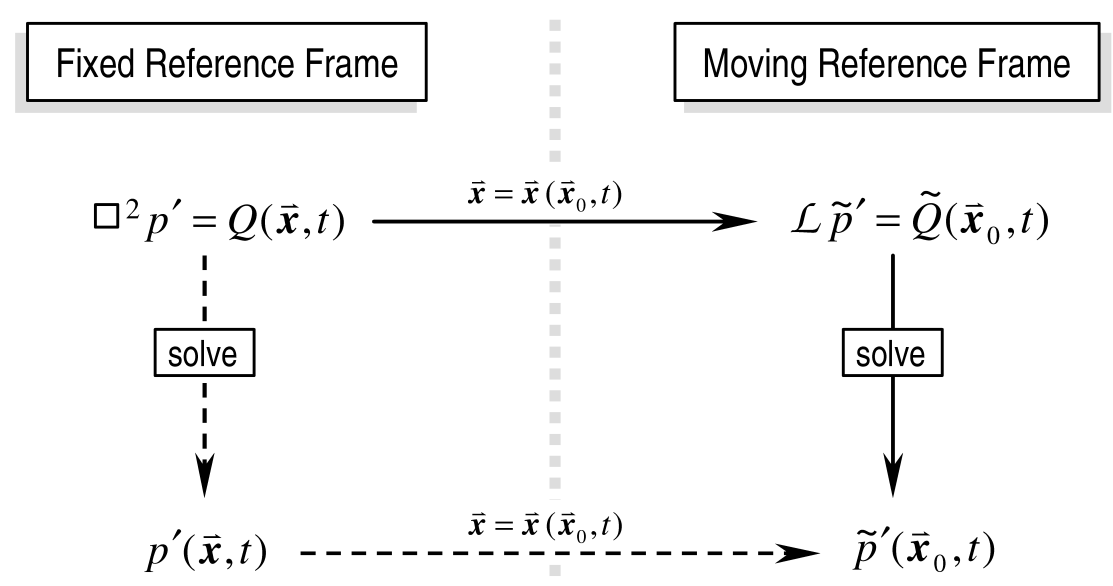

Fig. 1. Solution Diagram. The coordinate transformation can be incorporated before solving the equation (solid line) or after the solution is obtained (dashed line).

where $\mathcal{L}$ is a complicated wave-like operator, and $\tilde{Q}(\vec{x}, t)$ is the associated source term in the moving reference frame. In most cases, the solution of Eq. (1) is more easily obtained than the solution of Eq. (2) because of the simplicity of the free-space Green's function for the wave operator $\square^{2}$. Therefore, the second solution path is chosen, as shown by the dashed line in Fig. 1. For the problems under present consideration, the solution $p^{\prime}(\overrightarrow{\boldsymbol{x}}, t)$ in the fixed reference frame is readily available. The solution $\tilde{p}^{\prime}\left(\overrightarrow{\boldsymbol{x}}_{0}, t\right)$ in the moving reference frame is then obtained by applying a suitable coordinate transformation to the fixed-frame solution, i.e.,

$$
\tilde{p}^{\prime}\left(\overrightarrow{\boldsymbol{x}}_{0}, t\right)=p^{\prime}\left(\overrightarrow{\boldsymbol{x}}\left(\overrightarrow{\boldsymbol{x}}_{0}, t\right), t\right)
$$

For an appropriately chosen coordinate transformation, all of the solution's geometric terms in the moving reference frame become time independent, thus facilitating the subsequent statistical analysis. The required transformation $\overrightarrow{\boldsymbol{x}}=\overrightarrow{\boldsymbol{x}}\left(\overrightarrow{\boldsymbol{x}}_{0}, t\right)$ is easily specified for the cases under consideration in the present paper.

\section{A Wing in Uniform Rectilinear Subsonic Motion}

As early as 1954, Powell studied boundary layer noise from a rigid boundary as a distribution of fluctuating dipoles [10]. In 1960, he studied this problem further [11], extending, to some extent, the work of Curle [12]. In this section, the noise from random surface pressure fluctuations on a wing in motion is considered. Surface pressure fluctuations are caused either by the interaction of the wing with atmospheric turbulence 
or by turbulent fluctuations in the boundary layer. The acoustic pressure $p^{\prime}(\overrightarrow{\boldsymbol{x}}, t)$ can be calculated from the solution of the FW-H equation, retaining only the loading term:

$$
\square^{2} p^{\prime}=-\frac{\partial}{\partial x_{i}}\left[p n_{i} \delta(f)\right]
$$

Here, $\square^{2}$ denotes the linear wave operator with constant sound speed $c, p$ is the fluctuating pressure on the wing surface, and $\delta(\cdot)$ is the Dirac delta function. The wing surface is defined by the function $f(\overrightarrow{\boldsymbol{x}}, t)$ such that $f=0$ on the surface of the wing and $f>0$ in the region exterior to the surface. The notation $n_{i}$ represents the $i$-th component of the outward unit surface normal $\vec{n}=\vec{\nabla} f$. In this equation and henceforth, unless otherwise noted, the summation convention is assumed for repeated indices.

The solution of Eq. (4) for a wing in uniform motion is [13, 14]:

$$
4 \pi p^{\prime}(\overrightarrow{\boldsymbol{x}}, t)=\frac{1}{c} \int_{f=0}\left[\frac{\dot{p} n_{i} \hat{r}_{i}}{r\left(1-M_{r}\right)^{2}}\right]_{r e t} d S+\int_{f=0}\left[\frac{p\left[\left(1-M^{2}\right) n_{i} \hat{r}_{i}-\left(1-M_{r}\right) M \hat{r}_{1}\right]}{r^{2}\left(1-M_{r}\right)^{3}}\right]_{r e t} d S
$$

The flight velocity vector $\vec{V}=[V, 0,0]^{T}$ is directed along the $x_{1}$-axis. The observer space-time variables are denoted $(\overrightarrow{\boldsymbol{x}}, t)$ in the reference frame fixed to the medium at rest. However, it is important to remember that the surface integration is performed with respect to the spatial variables in the moving reference frame. The variable $\dot{p}$ is the time rate of change of the fluctuating surface pressure $p$ as measured by a transducer on the wing surface, and $\hat{r}_{i}$ is a component of the unit radiation vector $\hat{\boldsymbol{r}}=\overrightarrow{\boldsymbol{r}} / r$. The quantity $M_{r}$ is the Mach number in the radiation direction, defined by $M_{r}=\overrightarrow{\boldsymbol{M}} \cdot \hat{\boldsymbol{r}}$, where $\overrightarrow{\boldsymbol{M}}=\overrightarrow{\boldsymbol{V}} / \boldsymbol{c}$ is the Mach number vector based on the forward velocity of the wing. The subscript " $r e t$ " denotes evaluation of the bracketed terms at retarded time $t-r / c$. Note that the far field radiation is governed by the time rate of fluctuations of the surface pressure.

A frame of reference that moves with the wing is now considered (See Fig. 2). Let this moving reference frame coincide with the frame fixed to the undisturbed medium at the time $t=0$. To find the acoustic pressure at the observer position $\vec{x}_{0}$ in the moving frame, $\overrightarrow{\boldsymbol{x}}$ must be replaced with $\overrightarrow{\boldsymbol{x}}_{0}+\overrightarrow{\boldsymbol{V}} t$ in Eq. (5). If an arbitrary source point on the wing, in the moving frame of reference, is described by the position vector $\overrightarrow{\boldsymbol{\eta}}$, then the following two functions are time independent and can be written in terms of the variables $\overrightarrow{\boldsymbol{x}}_{0}$ and $\overrightarrow{\boldsymbol{\eta}}$ :

$$
F_{1}\left(\overrightarrow{\boldsymbol{x}}_{0}, \overrightarrow{\boldsymbol{\eta}}\right)=\left[\frac{n_{i} \hat{r}_{i}}{4 \pi c r\left(1-M_{r}\right)^{2}}\right]_{r e t}, \quad F_{2}\left(\overrightarrow{\boldsymbol{x}}_{0}, \overrightarrow{\boldsymbol{\eta}}\right)=\left[\frac{\left(1-M^{2}\right) n_{i} \hat{r}_{i}-\left(1-M_{r}\right) M \hat{r}_{1}}{4 \pi r^{2}\left(1-M_{r}\right)^{3}}\right]_{r e t}
$$

See [15, Sec. 5] for details of the analytic expressions for the two functions in Eq. (6) in terms of the variables $\overrightarrow{\boldsymbol{x}}_{0}$ and $\overrightarrow{\boldsymbol{\eta}}$. The time independence of these two functions greatly simplifies the statistical analysis of the acoustic pressure. Note that the emission distance 


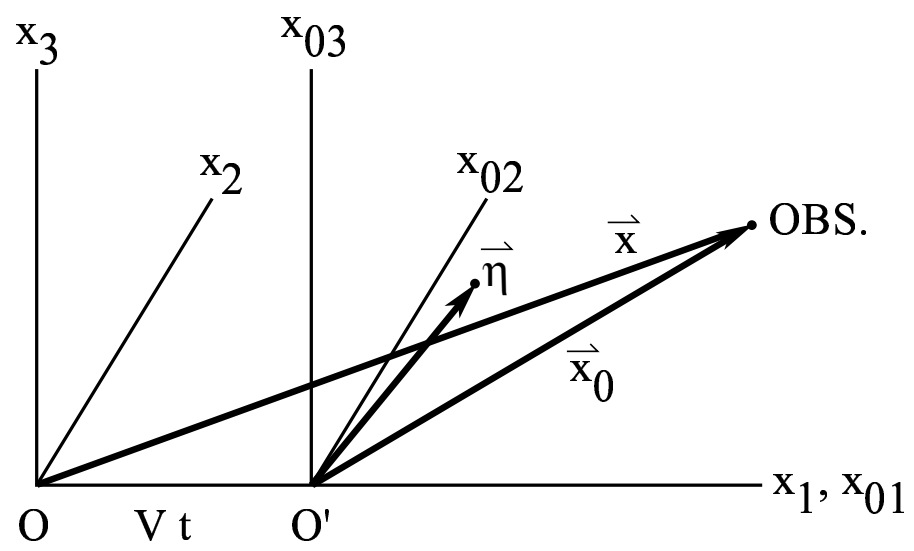

Fig. 2. The reference frames used in the analysis of a wing or a jet in uniform motion. The $\overrightarrow{\boldsymbol{x}}$-frame is fixed to the medium. The $\overrightarrow{\boldsymbol{x}}_{0}$-frame moves with the wing or the jet at speed $V$ along the $x_{1}$-axis. The leading edge of the wing faces in the positive $x_{1}$ direction. The line segment $\overline{00^{\prime}}$ has length $V t, \overrightarrow{\boldsymbol{\eta}}$ is the source position and $\overrightarrow{\boldsymbol{x}}_{0}$ is the observer position in the moving reference frame. For a propeller, the $\overrightarrow{\boldsymbol{x}}_{0}$-frame is attached to the propeller blade, it rotates around the $x_{1}$-axis, and moves forward with speed $V$ along the same axis.

$r^{*}=[r]_{\text {ret }}$, as well as other geometric variables such as $\hat{r}_{i}$, are also time independent and can be expressed as a function of $\overrightarrow{\boldsymbol{x}}_{0}$ and $\overrightarrow{\boldsymbol{\eta}}$, as discussed in [15, Sec. 5]. Fig. 3 shows the geometry associated with radiation from a source at position $\vec{\eta}$ in the moving frame. The pictured construction shows that this geometry is independent of time. The triangle $\mathrm{ABC}$, sometimes called the Garrick triangle in the literature of linear unsteady aerodynamics, is seen by an observer positioned in the stationary frame of reference. The shape and the dimensions of this triangle do not change with time.

Therefore, Eq. (5) can be rewritten with respect to the moving reference frame in the following simplified form:

$$
\begin{aligned}
\tilde{p}^{\prime}\left(\overrightarrow{\boldsymbol{x}}_{0}, t\right) & \equiv p^{\prime}\left(\overrightarrow{\boldsymbol{x}}_{0}+\overrightarrow{\boldsymbol{V}} t, t\right) \\
& =\int_{f=0}\left[F_{1}\left(\overrightarrow{\boldsymbol{x}}_{0}, \overrightarrow{\boldsymbol{\eta}}\right) \dot{p}\left(\overrightarrow{\boldsymbol{\eta}}, t-r^{*} / c\right)+F_{2}\left(\overrightarrow{\boldsymbol{x}}_{0}, \overrightarrow{\boldsymbol{\eta}}\right) p\left(\overrightarrow{\boldsymbol{\eta}}, t-r^{*} / c\right)\right] d S
\end{aligned}
$$

Using the notation $\langle\cdot\rangle$ to denote an ensemble average in time, the autocorrelation of the acoustic pressure in Eq. (7) can be written: 


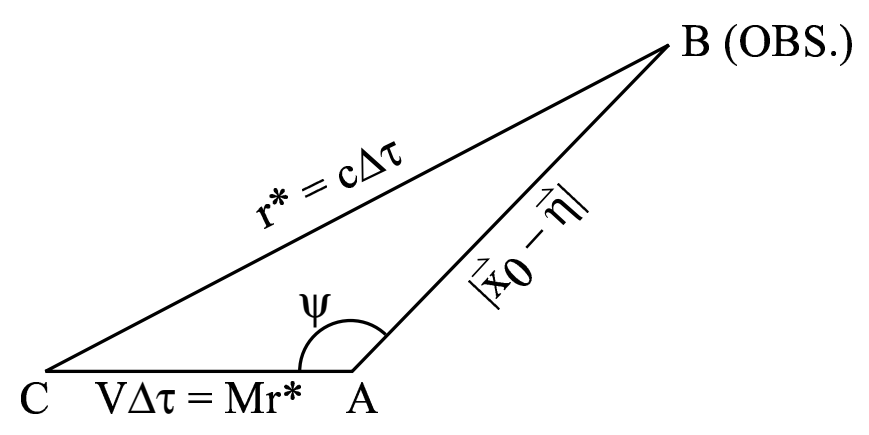

Fig. 3. The geometry of radiation as seen by an observer fixed to the medium at rest. $\mathrm{A}$ and $\mathrm{B}$ are the source and observer positions at a given observer time $t$. $\mathrm{C}$ is the emission position of the source, and $\overline{\mathrm{CB}}=c \Delta \tau$, where $\Delta \tau$ is the signal propagation time from $\mathrm{C}$ to $\mathrm{B}$. Application of the law of cosines to triangle ABC yields $r^{*}$ as a function of $\left(\overrightarrow{\boldsymbol{x}}_{0}, \overrightarrow{\boldsymbol{\eta}}\right)$, independent of time, as are $M_{r}$ and $\hat{r}_{i}$. Note that the symbol $\tau$ denotes source time in this figure, and not delay time.

$$
\begin{aligned}
\left\langle\tilde{p}^{\prime}\left(\overrightarrow{\boldsymbol{x}}_{0}, t\right) \tilde{p}^{\prime}\left(\overrightarrow{\boldsymbol{x}}_{0}, t+\tau\right)\right\rangle=\int_{f=0} \int_{f=0} & {\left[F_{1} F_{1}^{\prime}\left\langle\dot{p}\left(\overrightarrow{\boldsymbol{\eta}}, t-r^{*} / c\right) \dot{p}\left(\overrightarrow{\boldsymbol{\eta}}^{\prime}, t+\tau-r^{\prime *} / c\right)\right\rangle\right.} \\
& +F_{1} F_{2}^{\prime}\left\langle\dot{p}\left(\overrightarrow{\boldsymbol{\eta}}, t-r^{*} / c\right) p\left(\overrightarrow{\boldsymbol{\eta}}^{\prime}, t+\tau-r^{\prime *} / c\right)\right\rangle \\
& +F_{1}^{\prime} F_{2}\left\langle p\left(\overrightarrow{\boldsymbol{\eta}}, t-r^{*} / c\right) \dot{p}\left(\overrightarrow{\boldsymbol{\eta}}^{\prime}, t+\tau-r^{\prime *} / c\right)\right\rangle \\
& \left.+F_{2} F_{2}^{\prime}\left\langle p\left(\overrightarrow{\boldsymbol{\eta}}, t-r^{*} / c\right) p\left(\overrightarrow{\boldsymbol{\eta}}^{\prime}, t+\tau-r^{\prime *} / c\right)\right\rangle\right] d S_{\bar{\eta}^{\prime}} d S_{\vec{\eta}}
\end{aligned}
$$

where the prime notation on the quantities $F_{1}, F_{2}$, and $r^{*}$ denotes the replacement of the variable $\overrightarrow{\boldsymbol{\eta}}$ in these quantities with $\overrightarrow{\boldsymbol{\eta}}^{\prime}$.

Now, define a surface pressure cross-correlation function by

$$
R_{p p} \equiv\left\langle p(\overrightarrow{\boldsymbol{\eta}}, t) p\left(\overrightarrow{\boldsymbol{\eta}}^{\prime}, t+\tau\right)\right\rangle
$$

Using the ergodic hypothesis, the variable dependence of the cross-correlation function in Eq. (9) can be expressed:

$$
R_{p p} \equiv\left\langle p\left(\overrightarrow{\boldsymbol{\eta}}, t-r^{*} / c\right) p\left(\overrightarrow{\boldsymbol{\eta}}^{\prime}, t+\tau-r^{* *} / c\right)\right\rangle=R_{p p}\left(\overrightarrow{\boldsymbol{\eta}}, \overrightarrow{\boldsymbol{\eta}}^{\prime}, \tau+\left(r^{*}-r^{* *}\right) / c\right)
$$

Let $A(\overrightarrow{\boldsymbol{\eta}})$ denote the correlation area as a function of $\overrightarrow{\boldsymbol{\eta}}$. Then the acoustic-pressure correlation function in the moving reference frame can be written: 


$$
\begin{aligned}
& \left\langle\tilde{p}^{\prime}\left(\overrightarrow{\boldsymbol{x}}_{0}, t\right) \quad \tilde{p}^{\prime}\left(\overrightarrow{\boldsymbol{x}}_{0}, t+\tau\right)\right\rangle= \\
& \quad \int_{f=0} \int_{A(\vec{\eta})}\left[F_{1} F_{1}^{\prime} \partial_{\tau \tau} R_{p p}+\left(F_{1} F_{2}^{\prime}+F_{1}^{\prime} F_{2}\right) \partial_{\tau} R_{p p}+F_{2} F_{2}^{\prime} R_{p p}\right] d S_{\vec{\eta}^{\prime}} d S_{\bar{\eta}}
\end{aligned}
$$

where $\partial_{\tau}$ and $\partial_{\tau \tau}$ denote, respectively, the first and second partial derivatives with respect to delay time $\tau$. Eq. (11) is the main result of this section and it is exact for the calculation of both near and far field loading noise for a surface in uniform rectilinear motion.

A few remarks are in order at this point. Note that the cross-correlation function $R_{p p}$ in Eq. (9) can be obtained from measurements by transducers on the wing surface. Furthermore, although the assumption of ergodicity is reasonable, one cannot further assume that the dependence of $R_{p p}$ on the position variables $\overrightarrow{\boldsymbol{\eta}}$ and $\overrightarrow{\boldsymbol{\eta}}^{\prime}$ can be generally expressed in terms of the separation vector $\overrightarrow{\boldsymbol{\eta}}-\overrightarrow{\boldsymbol{\eta}}^{\prime}$. Finally, it is interesting that, in the far field, Eq. (11) takes the following simple form:

$$
\left\langle\tilde{p}^{\prime}\left(\overrightarrow{\boldsymbol{x}}_{0}, t\right) \tilde{p}^{\prime}\left(\overrightarrow{\boldsymbol{x}}_{0}, t+\tau\right)\right\rangle=\int_{f=0} \int_{A(\overrightarrow{\boldsymbol{\eta}})} F_{1} F_{1}^{\prime} \partial_{\tau \tau} R_{p p} d S_{\overline{\boldsymbol{\eta}}^{\prime}} d S_{\overline{\boldsymbol{\eta}}}
$$

However, because the functions $F_{1}$ and $F_{2}$ can easily be computed numerically, the restriction to the far field is not necessary, but merely a convenience for appropriate problems. If qualitative results are desired, e.g., a Mach number dependence rule for an observer in the far field, then further simplifying assumptions can be made in the above equation. It is clear from Eq. (11) that, for a realistic problem when the observer is in the near field, a Mach number dependence rule that is derived for a simple dipole in motion cannot be expected to hold. This is because of the complicated dependence of the functions in Eq. (11) on $\overrightarrow{\boldsymbol{x}}_{0}$ and $\overrightarrow{\boldsymbol{\eta}}$. However, numerical evaluation of the integral on the right-hand of Eq. (11) is quite feasible and can give a good picture of the near field behavior of the broadband noise from a wing in motion.

By analyzing experimental data, analytical models can be deduced for the surface pressure cross-correlation function on a wing. As in jet noise analysis, such models are very important. If available, the experimentally measured surface pressure crosscorrelation function can be directly used in a noise calculation. It is interesting to note that the numerical evaluation of the correlation function of the acoustic pressure in the near field is no more difficult than in the far field computation.

\section{A Propeller in Uniform Forward Flight}

In order to include the effects of rotational motion, the solution of Eq. (4) becomes slightly more complicated than the solution for the wing in Eq. (5). For a propeller with forward flight velocity $\overrightarrow{\boldsymbol{V}}$, the acoustic pressure at an observer location $\overrightarrow{\boldsymbol{x}}$ at time $t$ is given by the following expression $[13,14]$ : 


$$
\begin{aligned}
4 \pi p^{\prime}(\overrightarrow{\boldsymbol{x}}, t)= & \frac{1}{c} \int_{f=0}\left[\frac{\dot{p} n_{i} \hat{r}_{i}}{r\left(1-M_{r}\right)^{2}}+\frac{p \dot{M}_{i} \hat{r}_{i}}{r\left(1-M_{r}\right)^{3}}\right]_{r e t} d S \\
& +\int_{f=0}\left[\frac{p\left[\left(1-M^{2}\right) n_{i} \hat{r}_{i}-\left(1-M_{r}\right) M_{i} \hat{r}_{i}\right]}{r^{2}\left(1-M_{r}\right)^{3}}\right]_{r e t} d S
\end{aligned}
$$

where $\dot{M}_{i}$ is the $i$-th component of the local surface acceleration vector $\partial \vec{v} / \partial \tau$ divided by the sound speed $c$. The reader is again reminded that the surface integration is performed with respect to the variable $\overrightarrow{\boldsymbol{\eta}}$ in the moving reference frame. The objective in this section is to derive a result similar to Eq. (11) for the more complicated case of a rotating propeller in forward motion.

Note that the simplicity of Eq. (11) depends on the time independence of $F_{1}, F_{2}$, and $r^{*}$ in Eq. (7). It is natural to ask whether there exists a straightforward extension of Eq. (7) to rotational motion. If the observer is placed in a rotational reference frame that is attacheded to the propeller blade, can time independent functions $F_{1}, F_{2}$, and $r^{*}$ again be determined? The answer to this question is affirmative, by the following reasoning.

Let $\overrightarrow{\boldsymbol{\omega}}$ denote the angular velocity vector of the propeller and $\overrightarrow{\boldsymbol{V}}$ its uniform flight velocity. In a similar fashion to the wing problem above, assume that a reference frame that rotates with the propeller and a reference frame that is fixed to the medium at rest coincide at the time $t=0$. Let $\overrightarrow{\boldsymbol{x}}_{0}$ denote the observer position in the rotating reference frame and $\overrightarrow{\boldsymbol{\eta}}$ be the position vector of a point on the propeller blade surface in the same reference frame. Note that the relative position of these two vectors is the same for all observer time $t$. Within this framework, define the following two functions: ${ }^{\dagger}$

$$
\begin{aligned}
& F_{1}\left(\overrightarrow{\boldsymbol{x}}_{0}, \overrightarrow{\boldsymbol{\eta}}\right)=\left[\frac{n_{i} \hat{r}_{i}}{4 \pi c r\left(1-M_{r}\right)^{2}}\right]_{r e t} \\
& F_{2}\left(\overrightarrow{\boldsymbol{x}}_{0}, \overrightarrow{\boldsymbol{\eta}}\right)=\left[\frac{\dot{M}_{i} \hat{r}_{i}}{4 \pi c r\left(1-M_{r}\right)^{3}}\right]_{r e t}+\left[\frac{\left(1-M^{2}\right) n_{i} \hat{r}_{i}-\left(1-M_{r}\right) M_{i} \hat{r}_{i}}{4 \pi r^{2}\left(1-M_{r}\right)^{3}}\right]_{r e t}
\end{aligned}
$$

It remains to show that the two functions in Eq. (14), as well as $r^{*}$, are independent of the observer time $t$. An analogous result to Eq. (7) will then immediately follow.

The time independence of $F_{1}, F_{2}$, and $r^{*}$ can be demonstrated in the following way. Referring to Fig. 4, at a given observer time $t$, freeze the position of the observer in the reference frame fixed to the medium at rest. The contribution to the acoustic pressure at the observer position $\overrightarrow{\boldsymbol{x}}_{0}$ from a source position $\overrightarrow{\boldsymbol{\eta}}$ on the propeller blade surface requires the evaluation of the location of the source position at the retarded time $t-r^{*} / c$. The required location of $\overrightarrow{\boldsymbol{\eta}}$ can be visualized by letting the point $\overrightarrow{\boldsymbol{\eta}}$ trace its history backward

\footnotetext{
${ }^{\dagger}$ Eq. (14), as shown here, has been corrected from that printed on page 343 in International Journal of Aeroacoustics, Vol. 2, No. $3 \&$ 4, 2003. An erratum has been submitted to the journal.
} 


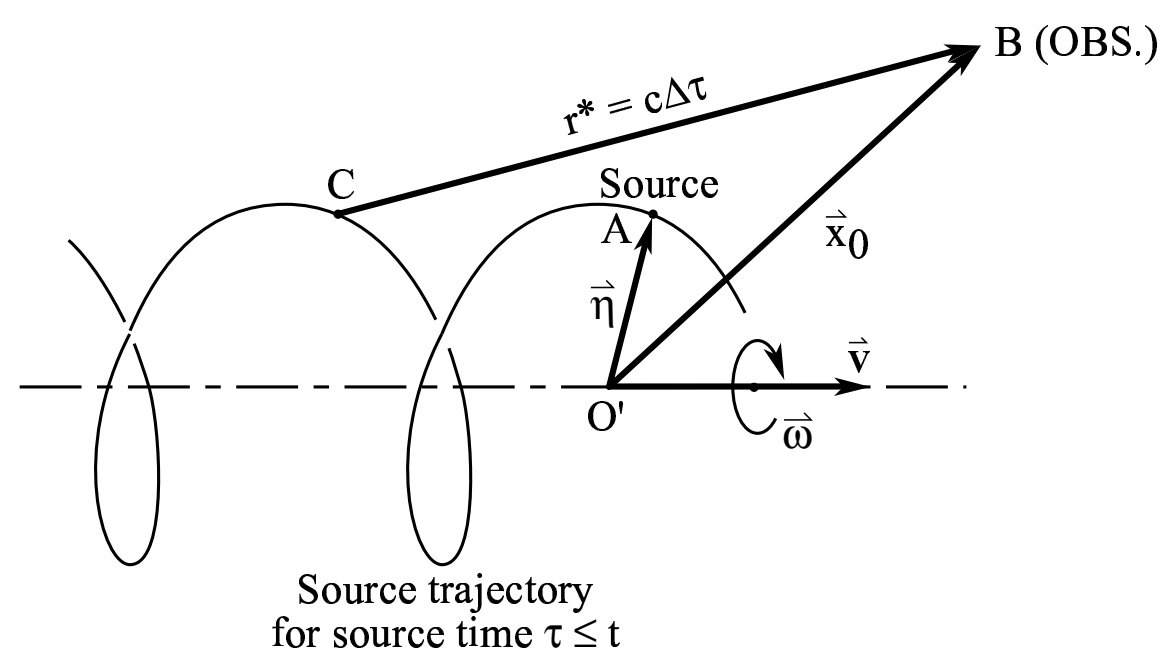

Fig. 4. The geometry of radiation from a source on a propeller blade, as seen by an observer fixed to the medium. A and B are the source and observer positions at a given observer time $t$. $\mathrm{C}$ is the emission position of the source, and $\overline{\mathrm{CB}}=c \Delta \tau$, where $\Delta \tau$ is the signal propagation time from $\mathrm{C}$ to $\mathrm{B}$. $\mathrm{C}$ is found from the relation $\overline{\mathrm{CA}}=$ (helical speed) $\times \Delta \tau=$ (helical Mach number) $\times r^{*}$, where $\overline{\mathrm{CA}}$ is the distance along the helix from $\mathrm{C}$ to A. This shows that $r^{*}, M_{r}$, and $\hat{r}_{i}$ are functions of $\left(\overrightarrow{\boldsymbol{x}}_{0}, \overrightarrow{\boldsymbol{\eta}}\right)$. Note that the symbol $\tau$ denotes source time in this figure, and not delay time.

in time on a helix (See Fig. 4). The emission point of this source lies on this helical path and is always in the same physical location relative to the observer position, independent of the observer time $t$. In this discussion, the observer is positioned in the frame fixed to the medium and observing the propagation phenomenon.

Using Eqs. (13) and (14) within the aforementioned framework of a rotating reference frame, the acoustic pressure at $\overrightarrow{\boldsymbol{x}}_{0}$ can be expressed in the following form:

$$
\begin{aligned}
\tilde{p}^{\prime}\left(\overrightarrow{\boldsymbol{x}}_{0}, t\right) & \equiv p^{\prime}\left(\overrightarrow{\boldsymbol{x}}_{0}+\left(\overrightarrow{\boldsymbol{\omega}} \times \overrightarrow{\boldsymbol{x}}_{0}+\overrightarrow{\boldsymbol{V}}\right) t, t\right) \\
& =\int_{f=0}\left[F_{1}\left(\overrightarrow{\boldsymbol{x}}_{0}, \overrightarrow{\boldsymbol{\eta}}\right) \dot{p}\left(\overrightarrow{\boldsymbol{\eta}}, t-r^{*} / c\right)+F_{2}\left(\overrightarrow{\boldsymbol{x}}_{0}, \overrightarrow{\boldsymbol{\eta}}\right) p\left(\overrightarrow{\boldsymbol{\eta}}, t-r^{*} / c\right)\right] d S
\end{aligned}
$$

where $F_{1}$ and $F_{2}$ are defined in Eq. (14). Note that, for a rotating propeller, the functions $F_{1}, F_{2}$, and $r^{*}$, as well as other geometric quantities, cannot be determined in closed form, even if there is no forward flight. However, these functions can easily be computed. 
Thus, it has been established that the right-hand sides of Eqs. (15) and (7) are identical in form, with time independent functions $F_{1}, F_{2}$, and $r^{*}$. Therefore, Eq. (11) is also valid for the acoustic-pressure correlation function at an observer in a rotating reference frame that is attached to the propeller blade, with the understanding that $F_{1}$ and $F_{2}$ are given by Eq. (14). Moreover, all of the comments following Eq. (11) for a wing in uniform motion also apply to a propeller in forward flight.

The measurement of sound by a rotating microphone presents some experimental difficulties, such as the presence of wind noise. However, it is possible to use advanced signal analysis techniques to overcome these difficulties. The results in this section are presented to bring attention to the simplicity of Eq. (11) for a rotating microphone.

\section{A Jet In Uniform Rectilinear Subsonic Motion}

A jet in uniform rectilinear subsonic motion is now considered. Lighthill's [8] jet noise equation is:

$$
\square^{2} p^{\prime}(\overrightarrow{\boldsymbol{x}}, t)=\frac{\partial^{2} T_{i j}}{\partial x_{i} x_{j}}
$$

where $T_{i j}$ is the Lighthill stress tensor. The jet is assumed free of shocks, the implications of which will be discussed below. Consider a frame of reference that moves with forward flight of the jet and moves with a uniform rectilinear subsonic velocity $\overrightarrow{\boldsymbol{V}}$. In [16], an exact solution of Eq. (16) is derived that involves only the observer time derivatives and includes the near field terms:

$$
\begin{aligned}
4 \pi p^{\prime}(\overrightarrow{\boldsymbol{x}}, t)=\frac{1}{c^{2}} \frac{\partial^{2}}{\partial t^{2}} \int_{\mathcal{V}} & {\left[\frac{T_{r r}}{r\left(1-\tilde{M}_{r}\right)}\right]_{r e t} d \overrightarrow{\boldsymbol{\eta}}+\frac{1}{c} \frac{\partial}{\partial t} \int_{\mathcal{V}}\left[\frac{3 T_{r r}-T_{i i}}{r^{2}\left(1-\tilde{M}_{r}\right)}\right]_{r e t} d \overrightarrow{\boldsymbol{\eta}} } \\
& +\int_{\mathcal{V}}\left[\frac{3 T_{r r}-T_{i i}}{r^{3}\left(1-\tilde{M}_{r}\right)}\right]_{r e t} d \overrightarrow{\boldsymbol{\eta}}
\end{aligned}
$$

Here, $T_{r r}=T_{i j} \hat{r}_{i} \hat{r}_{j}$ and $\tilde{M}_{r}$ is the Mach number in the direction of noise radiation based on the forward velocity $\vec{V}$. The variable $\mathcal{V}$ represents the turbulent volume in the jet while $d \overrightarrow{\boldsymbol{\eta}}$ denotes a volume element. It is important to note that the observer time derivatives here are performed while keeping the observer location $\overrightarrow{\boldsymbol{x}}$ fixed. In fact, it is reemphasized here that the observer in Eq. (17) is described in a reference frame that is fixed to the undisturbed medium. Furthermore, all the velocities in the Lighthill stress tensor terms are evaluated relative to the frame fixed to the undisturbed medium. However, the integrals in Eq. (14) are performed in the moving reference frame. These facts can all be inferred from the derivation of the above equation.

Brentner [17] has derived an equivalent expression to Eq. (17) when the time derivatives are taken inside the integrals: 


$$
\begin{aligned}
4 \pi p^{\prime}(\overrightarrow{\boldsymbol{x}}, t) & =\int_{\mathcal{V}}\left[\frac{\ddot{T}_{i j} \hat{r}_{i} \hat{r}_{j}}{c^{2} r\left(1-\tilde{M}_{r}\right)^{3}}\right]_{r e t} d \overrightarrow{\boldsymbol{\eta}} \\
& +\int_{\mathcal{V}}\left[-\frac{\dot{T}_{i i}}{c r^{2}\left(1-\tilde{M}_{r}\right)^{2}}-\frac{4 \dot{T}_{i j} \tilde{M}_{i} \hat{r}_{i}}{c r^{2}\left(1-\tilde{M}_{r}\right)^{3}}+\frac{3\left(1-\tilde{M}^{2}\right) \dot{T}_{i j} \hat{r}_{i} \hat{r}_{j}}{c r^{2}\left(1-\tilde{M}_{r}\right)^{4}}\right]_{r e t} d \overrightarrow{\boldsymbol{\eta}} \\
& +\int_{V}\left[\frac{2 T_{i j} \tilde{M}_{i} \tilde{M}_{j}-\left(1-\tilde{M}^{2}\right) T_{i i}}{r^{3}\left(1-\tilde{M}_{r}\right)^{3}}-\frac{6\left(1-\tilde{M}^{2}\right) T_{i j} \tilde{M}_{i} \hat{r}_{i}}{r^{3}\left(1-\tilde{M}_{r}\right)^{4}}+\frac{3\left(1-\tilde{M}^{2}\right) T_{i j} \hat{r}_{i} \hat{r}_{j}}{r^{3}\left(1-\tilde{M}_{r}\right)^{5}}\right]_{r e t} d \overrightarrow{\boldsymbol{\eta}}
\end{aligned}
$$

In Eq. (18), a dot over a symbol denotes a source time derivative, keeping the variable $\overrightarrow{\boldsymbol{\eta}}$ fixed, i.e. the time rate of change of the parameter as measured in the moving frame. The tilde notation $\tilde{M}$ represents a Mach number based on the forward velocity $\vec{V}$. The above equation, specialized to the problem of rotating blade noise, has been used successfully by Brentner [17] to compute high-speed helicopter rotor blade noise.

In a manner similar to the previous two sections, if the observer is in the moving frame at the position $\overrightarrow{\boldsymbol{x}}_{0}$, then many of the terms in Eq. (18) are time independent. The acoustic pressure in the moving frame is, therefore:

$$
\begin{aligned}
& \tilde{p}^{\prime}\left(\overrightarrow{\boldsymbol{x}}_{0}, t\right) \equiv p^{\prime}\left(\overrightarrow{\boldsymbol{x}}_{0}+\overrightarrow{\boldsymbol{V}} t, t\right) \\
&=\int_{\mathcal{V}}\left[F_{i j}\left(\overrightarrow{\boldsymbol{x}}_{0}, \overrightarrow{\boldsymbol{\eta}}\right) \ddot{T}_{i j}\left(\overrightarrow{\boldsymbol{\eta}}, t-r^{*} / c\right)+G_{i j}\left(\overrightarrow{\boldsymbol{x}}_{0}, \overrightarrow{\boldsymbol{\eta}}\right) \dot{T}_{i j}\left(\overrightarrow{\boldsymbol{\eta}}, t-r^{*} / c\right)+H_{i j}\left(\overrightarrow{\boldsymbol{x}}_{0}, \overrightarrow{\boldsymbol{\eta}}\right) T_{i j}\left(\overrightarrow{\boldsymbol{\eta}}, t-r^{*} / c\right)\right] d \overrightarrow{\boldsymbol{\eta}}
\end{aligned}
$$

where three time independent tensor functions are defined as follows:

$$
\begin{aligned}
& F_{i j}\left(\overrightarrow{\boldsymbol{x}}_{0}, \overrightarrow{\boldsymbol{\eta}}\right)=\left[\frac{\hat{r}_{i} \hat{r}_{j}}{4 \pi c^{2} r\left(1-\tilde{M}_{r}\right)^{3}}\right]_{r e t} \\
& G_{i j}\left(\overrightarrow{\boldsymbol{x}}_{0}, \overrightarrow{\boldsymbol{\eta}}\right)=\left[-\frac{\delta_{i j}}{4 \pi c r^{2}\left(1-\tilde{M}_{r}\right)^{2}}-\frac{\tilde{M}_{i} \hat{r}_{i}}{\pi c r^{2}\left(1-\tilde{M}_{r}\right)^{3}}+\frac{3\left(1-\tilde{M}^{2}\right) \hat{r}_{i} \hat{r}_{j}}{4 \pi c r^{2}\left(1-\tilde{M}_{r}\right)^{4}}\right]_{r e t} \\
& H_{i j}\left(\overrightarrow{\boldsymbol{x}}_{0}, \overrightarrow{\boldsymbol{\eta}}\right)=\left[\frac{2 \tilde{M}_{i} \tilde{M}_{j}-\left(1-\tilde{M}^{2}\right) \delta_{i j}}{4 \pi r^{3}\left(1-\tilde{M}_{r}\right)^{3}}-\frac{3\left(1-\tilde{M}^{2}\right) \tilde{M}_{i} \hat{r}_{i}}{2 \pi r^{3}\left(1-\tilde{M}_{r}\right)^{4}}+\frac{3\left(1-\tilde{M}^{2}\right) \hat{r}_{i} \hat{r}_{j}}{4 \pi r^{3}\left(1-\tilde{M}_{r}\right)^{5}}\right]_{r e t}
\end{aligned}
$$

Here, $\delta_{i j}$ is the Kronecker delta. As in the previous two sections, $r^{*}=[r]_{r e t}$ is also time independent.

The velocity terms in the Reynolds stress term of the Lighthill quadrupole tensor are now considered. Let $u_{i}$ and $U_{i}$ denote the velocity components of the jet in the moving and the stationary frames, respectively. Then, the terms involving the velocity product in the Reynolds stress tensor in all the above equations must be $\rho U_{i} U_{j}$, as explained above. In terms of the velocity components in the moving frame, using the relation $U_{i}=u_{i}+V \delta_{1 i}$, the velocity terms of the Reynolds stress are: 


$$
\begin{array}{ll}
U_{1} U_{1}=u_{1} u_{1}+2 V u_{1}+V^{2} \\
U_{1} U_{i}=u_{1} u_{i}+V u_{i} \quad(i \neq 1) \\
U_{i} U_{j}=u_{i} u_{j} \quad(i, j \neq 1)
\end{array}
$$

Thus, the acoustic pressure in the moving frame can now be expressed in terms of quantities that can be measured in the moving frame of reference. Furthermore, the only time dependent quantities in Eq. (19) are the Lighthill stress tensor term and its time derivatives.

The analysis for the wing and the propeller in forward motion is now repeated. The autocorrelation function of the acoustic pressure can be expressed as follows:

$$
\begin{aligned}
\left\langle\tilde{p}^{\prime}(\overrightarrow{\boldsymbol{x}}, t) \tilde{p}^{\prime}(\overrightarrow{\boldsymbol{x}}, t+\tau)\right\rangle=\int_{\mathcal{V}} \int_{\mathcal{V}} & {\left[F_{i j} F_{k l}^{\prime}\left\langle\ddot{T}_{i j}\left(\overrightarrow{\boldsymbol{\eta}}, t-r^{*} / c\right) \ddot{T}_{k l}\left(\overrightarrow{\boldsymbol{\eta}}^{\prime}, t+\tau-r^{*} / c\right)\right\rangle\right.} \\
& +F_{i j} G_{k l}^{\prime}\left\langle\ddot{T}_{i j}\left(\overrightarrow{\boldsymbol{\eta}}, t-r^{*} / c\right) \dot{T}_{k l}\left(\overrightarrow{\boldsymbol{\eta}}^{\prime}, t+\tau-r^{* *} / c\right)\right\rangle \\
& +F_{i j} H_{k l}^{\prime}\left\langle\ddot{T}_{i j}\left(\overrightarrow{\boldsymbol{\eta}}, t-r^{*} / c\right) T_{k l}\left(\overrightarrow{\boldsymbol{\eta}}^{\prime}, t+\tau-r^{* *} / c\right)\right\rangle \\
& +G_{i j} F_{k l}^{\prime}\left\langle\dot{T}_{i j}\left(\overrightarrow{\boldsymbol{\eta}}, t-r^{*} / c\right) \ddot{T}_{k l}\left(\overrightarrow{\boldsymbol{\eta}}^{\prime}, t+\tau-r^{\prime *} / c\right)\right\rangle \\
& +G_{i j} G_{k l}^{\prime}\left\langle\dot{T}_{i j}\left(\overrightarrow{\boldsymbol{\eta}}, t-r^{*} / c\right) \dot{T}_{k l}\left(\overrightarrow{\boldsymbol{\eta}}^{\prime}, t+\tau-r^{\prime *} / c\right)\right\rangle \\
& +G_{i j} H_{k l}^{\prime}\left\langle\dot{T}_{i j}\left(\overrightarrow{\boldsymbol{\eta}}, t-r^{*} / c\right) T_{k l}\left(\overrightarrow{\boldsymbol{\eta}}^{\prime}, t+\tau-r^{* *} / c\right)\right\rangle \\
& +H_{i j} F_{k l}^{\prime}\left\langle T_{i j}\left(\overrightarrow{\boldsymbol{\eta}}, t-r^{*} / c\right) \ddot{T}_{k l}\left(\overrightarrow{\boldsymbol{\eta}}^{\prime}, t+\tau-r^{* *} / c\right)\right\rangle \\
& +H_{i j} G_{k l}^{\prime}\left\langle T_{i j}\left(\overrightarrow{\boldsymbol{\eta}}, t-r^{*} / c\right) \dot{T}_{k l}\left(\overrightarrow{\boldsymbol{\eta}}^{\prime}, t+\tau-r^{* *} / c\right)\right\rangle \\
& \left.+H_{i j} H_{k l}^{\prime}\left\langle T_{i j}\left(\overrightarrow{\boldsymbol{\eta}}, t-r^{*} / c\right) T_{k l}\left(\overrightarrow{\boldsymbol{\eta}}^{\prime}, t+\tau-r^{*} / c\right)\right\rangle\right] d \overrightarrow{\boldsymbol{\eta}} d \overrightarrow{\boldsymbol{\eta}}^{\prime}
\end{aligned}
$$

where the variable $\overrightarrow{\boldsymbol{\eta}}$ is replaced by the variable $\overrightarrow{\boldsymbol{\eta}}^{\prime}$ in the primed quantities. Now, define the cross-correlation function of the Lighthill stress tensor by:

$$
R_{i j k l}\left(\overrightarrow{\boldsymbol{\eta}}, \overrightarrow{\boldsymbol{\eta}}^{\prime}, \tau\right)=\left\langle T_{i j}(\overrightarrow{\boldsymbol{\eta}}, t) T_{l k}\left(\overrightarrow{\boldsymbol{\eta}}^{\prime}, t+\tau\right)\right\rangle
$$

Then, if $\mathcal{V}_{\mathrm{C}}(\overrightarrow{\boldsymbol{\eta}})$ is the correlation volume, Eq. (22) can be written as: 


$$
\begin{aligned}
&\left\langle\tilde{p}^{\prime}(\overrightarrow{\boldsymbol{x}}, t) \tilde{p}^{\prime}(\overrightarrow{\boldsymbol{x}}, t+\tau)\right\rangle=\int_{\mathcal{V}} \int_{\mathcal{V}_{c}(\overrightarrow{\boldsymbol{\eta}})}\left[F_{i j} F_{k l}^{\prime} \partial_{\tau \tau \tau} R_{i j k l}\left(\overrightarrow{\boldsymbol{\eta}}, \overrightarrow{\boldsymbol{\eta}}^{\prime}, \tau+r^{*} / c-r^{* *} / c\right)\right. \\
&+\left(F_{i j} G_{k l}^{\prime}+G_{i j} F_{k l}^{\prime}\right) \partial_{\tau \tau \tau} R_{i j k l}\left(\overrightarrow{\boldsymbol{\eta}}, \overrightarrow{\boldsymbol{\eta}}^{\prime}, \tau+r^{*} / c-r^{\prime *} / c\right) \\
&+\left(G_{i j} G_{k l}^{\prime}+F_{i j} H_{k l}^{\prime}+H_{i j} F_{k l}^{\prime}\right) \partial_{\tau \tau} R_{i j k l}\left(\overrightarrow{\boldsymbol{\eta}}, \overrightarrow{\boldsymbol{\eta}}^{\prime}, \tau+r^{*} / c-r^{\prime *} / c\right) \\
&+\left(G_{i j} H_{k l}^{\prime}+H_{i j} G_{k l}^{\prime}\right) \partial_{\tau} R_{i j k l}\left(\overrightarrow{\boldsymbol{\eta}}, \overrightarrow{\boldsymbol{\eta}}^{\prime}, \tau+r^{*} / c-r^{* *} / c\right) \\
&\left.+H_{i j} H_{k l}^{\prime} R_{i j k l}\left(\overrightarrow{\boldsymbol{\eta}}, \overrightarrow{\boldsymbol{\eta}}^{\prime}, \tau+r^{*} / c-r^{\prime *} / c\right)\right] d \overrightarrow{\boldsymbol{\eta}}^{\prime} d \overrightarrow{\boldsymbol{\eta}}
\end{aligned}
$$

where $\partial_{\tau}, \partial_{\tau \tau}$, etc., denote partial derivatives with respect to delay time $\tau$. For far field radiation with the observer in the moving frame, the autocorrelation of the acoustic pressure assumes the following simple form:

$$
\left\langle\tilde{p}^{\prime}(\overrightarrow{\boldsymbol{x}}, t) \quad \tilde{p}^{\prime}(\overrightarrow{\boldsymbol{x}}, t+\tau)\right\rangle=\int_{\mathcal{V}} \int_{\mathcal{V}_{\mathrm{c}}(\vec{\eta})}\left[F_{i j} F_{k l}^{\prime} \partial_{\tau \tau \tau \tau} R_{i j k l}\left(\overrightarrow{\boldsymbol{\eta}}, \overrightarrow{\boldsymbol{\eta}}^{\prime}, \tau+r^{*} / c-r^{\prime *} / c\right)\right] d \overrightarrow{\boldsymbol{\eta}}^{\prime} d \overrightarrow{\boldsymbol{\eta}}
$$

Therefore, to calculate the autocorrelation of the acoustic pressure in the moving frame, the cross-correlation function of the Lighthill stress tensor $R_{i j k l}\left(\overrightarrow{\boldsymbol{\eta}}, \overrightarrow{\boldsymbol{\eta}}^{\prime}, \tau\right)$ must be modeled, based on experimental observations. In general, such modeling is a difficult task. However, the mathematical framework given here can lead to the development of analytical models that can be used to make predictions to compare with acoustic measurements [18].

Note that, although Eq. (17) is valid for a jet with shocks, Eqs. (18) and (19) are only valid for shock-free jets. The reason for this restriction is that the observer time derivatives in Eq. (17) must be treated as generalized derivatives in order to interchange those derivatives with the integration $[19,20]$. When the generalized derivatives are brought inside the integral, any shock discontinuity in a jet will produce additional terms that are not included in the present jet noise analysis [21]. The analysis of jets with imbedded shocks is more complicated than what is presented here.

Finally, note that the results presented in this section are only a preliminary statistical analysis for jet noise. The works of Ribner [22], Ffowcs Williams [23], and others can be used in modeling the cross-correlation terms in Eq. (24).

\section{Concluding Remarks}

Some exact analytical results have been derived for the prediction of broadband noise radiated from a wing, a propeller, and a jet in uniform subsonic motion. For an observer in an appropriate frame of reference, the correlation function of the acoustic pressure can be directly related to the space-time cross-correlation of either, the surface pressure on a wing or propeller blade, or the cross-correlation of the Lighthill stress tensor in the case of a jet. It has been shown that the inclusion of the near field terms does not add to the complexity of the analysis. The development of these results requires that the observer be in the moving reference frame in the case of a wing or a jet in forward flight, or in a 
rotating reference frame in the propeller case. These requirements introduce many time independent functions in the integrands of the acoustic formulations, resulting in considerable simplification of the statistical analysis.

These analytical results were developed with practical applications in mind. In particular, the goal of the present analytical development is to obtain statistical formulations for which the required input data are not unusual or difficult to measure. It is shown here that this can be achieved by the selection of an appropriate frame of reference in which the observer position is described. Although some of the present results may appear complicated, they are conceptually simple for numerical work. One of the avenues open to current researchers involves experimenting with analytical modeling of the cross-correlation function in acoustic formulations, using measured data for guidance. In addition, experimental data can always be used directly in the present analytical results, when they are available.

As a final remark, note that an analysis similar to that presented herein can be used to obtain a cross-correlation function that correlates the surface pressure to the acoustic pressure. The derivation of such a cross-correlation function was presented by Siddon [4]. As reported by Siddon, such an analysis can be very useful for the identification of noise generating regions.

The aeroacoustics community owes much to pioneering researchers like Alan Powell, who led the rest of us to the importance of identifying and solving significant acoustic problems. We wish him all the best for many years to come.

\section{References}

1. A. Powell: On the Aerodynamic Noise of a Rigid Flat Plate Moving at Zero Incidence, J. Acoust. Soc. Am., 31(12), 1959, pp. 1649-1653.

2. H. H. Hubbard (Editor): Aeroacoustics of Flight Vehicles - Theory and Practice, Volume 1, Noise Sources, Acoustical Society of America, 1995.

3. W. C. Meecham: Surface and Volume Sound From Boundary Layers, J. Acoustical Society of America, 37(2), 1965, pp. 516-522.

4. T. E. Siddon: Surface Dipole Strength by Cross-Correlation Method, J. Acoustical Society of America, 53(2), 1973, pp. 619-633.

5. W. C. Meecham: Theory of Air Frame Noise: J. Acoustical Society of America, 57(6), Part II, 1975, pp. 1416-1420.

6. W. R. Miller and William C. Meecham: Large Scale Model Measurements of Airframe Noise Using Cross-Correlation Technique, J. Acoustical Society of America, 71(3), 1982, pp. 591-599.

7. T. F. Brooks and W. M. Humphreys: Flap-Edge Aeroacoustic Measurements and Predictions, J. of Sound and Vibration, 261, 2003, pp. 31-74.

8. M. J. Lighthill: On Sound Generated Aerodynamically. I. General Theory, Proceedings of the Royal Society of London, A 211, 1952, pp. 564 - 587. 
9. J. E. Ffowcs Williams and D. L. Hawkings: Sound Generation by Turbulence and Surfaces in Arbitrary Motion, Philosophical Transactions of the Royal Society, A 264, 1969, pp. $321-342$.

10. A. Powell: Thoughts on Boundary Layer Noise, Aeronautical Research Council (Great Britain) Report A.R.C. 16727, F.M. 2061, April 1954.

11. A. Powell: Aerodynamic Noise and the Plane Boundary, J. Acoust. Soc. Am., 32(8), 1960, pp. 982-990.

12. N. Curle: The Influence of Solid Boundaries on Aerodynamic Sound, Proc. Roy. Soc. (London), A231, 1955, pp. 505-514.

13. F. Farassat and G. P. Succi: The Prediction of Helicopter Rotor Discrete Frequency Noise, Vertica, 7(4), 1983, pp. 309-320.

14. K. S. Brentner: Prediction of Helicopter Rotor Discrete Frequency Noise- A computer program incorporating realistic blade motions and advanced acoustic formulation, NASA Technical Memorandum 87721, October 1986.

15. J. Casper and F. Farassat: A New Time Domain Formulation for Broadband Noise Prediction, Int. J. of Aeroacoustics, 1(3), 2002, pp. 207-240.

16. F. Farassat and K. S. Brentner: The Uses and Abuses of the Acoustic Analogy in Helicopter Rotor Noise Prediction, J. American Helicopter Society, Vol. 33, 1988, pp. 29-36.

17. K. S. Brentner: An Efficient and Robust Method for Predicting Helicopter Highspeed Impulsive Noise, J. Sound \& Vibration, 1997, 203(1), pp. 87-100.

18. P. J. Morris and F. Farassat: Acoustic Analogy and Alternative Theories for Jet Noise Prediction AIAA J., 40(4), April 2002, pp. 671-680.

19. I. M. Gelfand and G. E. Shilov: Generalized Functions: Properties and Operations, Volume 1, Academic Press, New York 1964.

20. F. Farassat: Introduction to Generalized Functions With Applications in Aerodynamics and Aeroacoustics, NASA Technical Paper 3428, 1994 (Corrected April 1996), Available at: http://techreports.larc.nasa.gov/ltrs/PDF/tp3428.pdf

21. F. Farassat and M. K Myers: An Analysis of the Quadrupole Noise Source of High Speed Rotating Blades, Computational Acoustics - Scattering, Gaussian Beams, and Aeroacoustics, D. Lee, A. Cakmak, R Vichnevetsky, Editors, Vol. 2, pp. 227-240, 1990.

22. H. S. Ribner: Theory of Two-Point Correlations of Jet Noise, NASA Technical Note, TND-8330, 1976.

23. J. E. Ffowcs Williams: The Noise from Turbulence Convected at High Speed, Philosophical Transactions of the Royal Society of London, Vol. 255, 1964, pp. 469503. 